\title{
SC-GAN: 3D self-attention conditional GAN with spectral normalization for multi-modal neuroimaging synthesis
}

4

\section{Authors}

Haoyu Lan a, the Alzheimer Disease Neuroimaging Initiative ${ }^{1}$, Arthur W Toga a, b, Farshid Sepehrband ${ }^{a, b}$

\section{Affiliation}

a) USC Stevens Neuroimaging and Informatics Institute, USC Keck School of Medicine, University of Southern California, Los Angeles CA

b) Alzheimer's Disease Research Center, Keck School of Medicine, University of Southern California, Los Angeles, CA, USA

\section{Correspondence to}

Farshid Sepehrband, PhD

USC Stevens Neuroimaging and Informatics Institute

USC Keck School of Medicine

University of Southern California

Email: farshid.sepehrband@loni.usc.edu

Contact: (323) 442-0148

\section{keyword}

Self-attention, spectral normalization, 3D GAN, MRI, PET, Synthesis

Number of Figures: 10

Number of Tables: 2

Word count: 5400

\footnotetext{
${ }^{1}$ Data used in preparation of this article were obtained from the Alzheimer's Disease Neuroimaging Initiative (ADNI) database (www.adni.loni.usc.edu). As such, the investigators within the ADNI contributed to the design and implementation of ADNI and/or provided data but did not participate in analysis or writing of this report. A complete listing of ADNI investigators can be found at:

http://adni.loni.usc.edu/wp-content/uploads/how to apply/ADNI_Acknowledgement List.pdf.
} 


\section{Abstract}

2

3 Image synthesis is one of the key applications of deep learning in neuroimaging, which enables

4 shortening of the scan time and/or improve image quality; therefore, reducing the imaging cost

5 and improving patient experience. Given the multi-modal and large-scale nature of neuroimaging

6 data, the synthesis task is computationally challenging. 2D image synthesis networks do not take

7 advantage of multi-dimensional spatial information and the 3D implementation has

8 dimensionality problem, negatively affecting the network reliability. These limitations hinder the

9 research and clinical applicability of deep learning-based neuroimaging synthesis. In this paper,

10 we proposed a new network that is designed and optimized for the application of multi-modal

$113 D$ synthesis of neuroimaging data. The network is based on 3D conditional generative

12 adversarial network (GAN), and employs spectral normalization and feature matching to stabilize

13 the training process and ensure optimization convergence. We also added a self-attention

14 module to model relationships between widely separated voxels. The performance of the

15 network was evaluated by predicting positron emission tomography (PET) images, Fractional

16 anisotropy (FA) and mean diffusivity (MD) maps from multi-modal magnetic resonance images

17 (MRI) of 265 and 497 individuals correspondingly. The proposed network, called self-attention

18 conditional GAN (SC-GAN), significantly outperformed conventional 2D conditional GAN and the

19 3D implementation, enabling robust 3D deep learning-based neuroimaging synthesis.

21 Keywords: 3D GAN, MRI, PET, image synthesis, self-attention, spectral normalization 


\section{Introduction}

2 Medical image synthesis is a technique to generate new parametric images from other 3 medical image modalities that contain a degree of similarity or mutual information. Medical

4 image synthesis can be used for a number of valuable applications, including shortening imaging 5 time, data augmentation, enabling low dose contrast administration and even image 6 enhancement (Hiasa et al., 2018; Nie et al., 2017; Roy et al., 2014; Shin et al., 2018; Wang et al., 7 2018; Yi et al., 2019). For the last five years, the power of image synthesis has been proven in 8 computer vision and image processing fields. In particular, generative adversarial networks (GAN) 9 (Goodfellow et al., 2014) has been shown to be an effective and reliable technique for image 10 synthesis (Huang et al., 2018). Variants of GAN like Conditional GAN (Mirza and Osindero, 2014) 11 and Cycle GAN (Zhu et al., 2017) also have been proposed to generalize GAN to different tasks and circumstances, including medical image synthesis.

Medical image synthesis with deep convolutional neuronal networks are often implemented using encoder-decoder networks, GAN or its variants. For example, Nie et al (Nie et al., 2018) proposed a deep convolutional adversarial network to synthesize Computer Tomography (CT)

17 images from magnetic resonance images (MRI), and also to synthesize 7T MRI from 3T images. Chen et al (Chen et al., 2019) implemented encoder-decoder convolutional neural network to synthesis positron emission tomography (PET) from ultra-low dose PET and MRI. Ouyang et al (Ouyang et al., 2019) used conditional GAN with task specific perceptual loss to synthesize PET

21 from ultra-low dose PET. These techniques used 2-dimensional (2D) or 2.5D network

22 implementations. For a 2D network implementation, image slices along one of 3D anatomical

23 planes (i.e. axial, coronal and sagittal) are trained independently and then combined or 24 ensembled in decoding step. Employing a 2D approach on a 3D data is suboptimal and inefficient 25 because it does not incorporate the 3D spatial information in the image, and/or it requires 26 multiple independent implementation of a same network along different image axes.

$3 \mathrm{D}$ networks were proposed to address the limitations of the $2 \mathrm{D}$ and $2.5 \mathrm{D}$ networks for the purpose of image synthesis. Wang et al (Wang et al., 2019) proposed a 3D conditional GAN 
1 network for PET synthesis from low dose input, which resulted to improved network 2 performance in comparison with the 2D implementation. Liu et al (Liu, 2019) showed that 3D

3 GAN performance can be improved further by incorporating an attention gate module to 4 generate synthesis results, which they used as the input of a segmentation task. Given that the 5 aim of image synthesis is to generate a new image from the existing images of the same individual, we anticipate that a self-attention module could further improve the performance of GAN. The 3D implementation of self-attention GAN however, with no specific modification/addition to network elements and optimizers, creates inconsistency problem due to the large differences of

9 feature distributions (Wang et al., 2019), negatively affecting the network reliability and sometimes fails to converge. In order to improve GAN performance and to address these

11 limitations, we developed a new 3D GAN and optimized it for neuroimaging synthesis.

Proposed 3D Self-attention Conditional GAN (SC-GAN) is constructed as follow: First, we extended 2D conditional GAN into 3D conditional GAN. Then, we added 3D self-attention module to 3D conditional GAN to generate 3D images with preserved brain structure and reduced blurriness within the synthesized images. We also introduced spectral normalization (Miyato et al., 2018), feature matching loss (Wang et al., 2018) and brain area root mean square error (RMSE) loss to stabilize training and prevent overfitting. SC-GAN is an end-to-end medical image synthesis network that can be applied on high-resolution input images (e.g. 256 × 256 × 256). SCGAN can also be applied on multi-modal input data and is designed using 3D convolutional layers.

The novelties and contributions of this technique are as follows.

23 I. For the first time, combining 3D self-attention module into 3D conditional GAN to generate high accuracy synthesis results with stable training process. A smooth training was achieved by using a series of stabilization techniques and modified loss function.

II. SC-GAN was tested on multiple datasets across different synthesis tasks and enables multi-model input, which can be generalized to other image synthesis applications.

III. SC-GAN source code is made available at https://github.com/Haoyulance/SC-GAN 


\section{Theory and Method}

2 Here we introduce the 3D Self-attention Conditional GAN (SC-GAN) theory and the mathematical

3 formulation of its components.

\section{B conditional GAN}

6 For the main body of the SC-GAN, we used conditional GAN, which is shown to be the optimum

7 choice of GAN for medical image synthesis and reconstruction with paired images (Wang et al.,

8 2019)(Ouyang et al., 2019)(Li et al., 2020)(Zhao et al., 2020). SC-GAN was then designed by adding

9 additional modules to conditional GAN that are described in detail in the next sections. This section describes the conditional GAN, which was also used as the baseline for evaluating the SC-

11 GAN.

13 In an unconditional GAN (Goodfellow et al., 2014), the generator learns the mapping from the

14 latent space to target image space by adversarial learning to generate the fake outcome, without 15 any label specify. Conditional GAN on the other hand learns to generate the outcome using a 16 specific condition, allowing the application of supervised learning for image-to-image generation.

17 Therefore, when ground truth data is available for training, conditional GAN is a powerful 18 network to do image translation. Conditional GAN uses below loss function:

$L_{c G A N}(G, D)=\mathbb{E}_{(x, y)}[\log D(x, y)]+\mathbb{E}_{(x, z)}[\log (1-D(x, G(x, z)))]$

22 where $x$ is the input image and also the condition image, $y$ is the ground truth image and $z$ is the

23 Gaussian noise. Notice $z$ is the sample in the latent space for unconditional GAN to generate

24 stochastic results. As for image to image translation, condition image $x$ has enough variance so 25 that generator would easily learn to ignore $z$ (Isola et al., 2017), (Ouyang et al., 2019). Therefore, 26 in conditional GAN noise $z$ is no longer provided to the generator and the loss function is 27 formulated as:

28

29

$L_{c G A N}(G, D)=\mathbb{E}_{(x, y)}[\log D(x, y)]+\mathbb{E}_{(x)}[\log (1-D(x, G(x)))]$ 
2 We adopted pix2pix (Isola et al., 2017), a variant network of 2D conditional GAN, as the network

3 structure of 3D conditional GAN. in our experiment, 3D conditional GAN has 8 layers generator,

4 similar to U-net (Ronneberger et al., 2015), and uses PatchGAN classifier(Isola et al., 2017) as the

5 discriminator. The objective function is:

$7 \min _{G}\left(\max _{D} L_{C G A N}(G, D)+\mu L_{1}(G)\right)$

9 Where $L_{1}(G)=\mathbb{E}_{(x, y)}\left(\|y-G(x)\|_{1}\right)$ is the $L_{1}$ loss between the ground truth and generated image and $\mu$ is the regularization term for the $L_{1}$ loss.

12 Generator's optimization aims to minimize the objective function. Only generator's weights are

13 updated in each iteration of the optimization. Discriminator's optimization aims to maximize the

14 objective function and therefore only discriminator's weights are updated in each iteration.

15 Generator and discriminator forward and backward propagate alternately till the training process reaches Nash equilibrium and network converges (Nash, 1950).

Feature matching loss

19 To stabilize the training, we incorporated a feature matching loss (Wang et al., 2018). Feature 20 matching loss is described as follow:

24 where $D^{i}$ is the $i_{t h}$ layer's feature map; $T$ is the total number of layers of discriminator and $N_{i}$ is 25 the number of elements in $i_{t h}$ layer's feature map.

27 Feature matching loss was added only to the generator loss, because only the $L_{F M}$ is required to 28 be minimized at generator's optimization. The objective function with feature matching loss is: 
$1 \min _{G}\left(\max _{D} L_{C G A N}(G, D)+\mu L_{1}(G)+\lambda L_{F M}(G, D)\right)$

2

3 where regularization term $(\lambda)$ controls the importance of the feature matching loss.

4

5 Brain area RMSE loss

6 Error calculation was done on brain voxels and the background was excluded. We calculated root

7 mean square error (RMSE) between masked $G$ and masked $y$, then added the RMSE to the

8 generator loss. We obtained the brain area $\left(\right.$ mask $\left._{y}\right)$ from the ground truth $y$, then, which was

9 used to calculate brain area RMSE (B-rmse) loss:

10

$11 \quad L_{B-r m s e}(G)=\sqrt{\frac{1}{N} \sum_{i=1}^{N}\left(\operatorname{mask}_{y}(y)^{i}-\operatorname{mask}_{y}(G(x))^{i}\right)^{2}}$

12

13 where $\operatorname{mask}_{y}(y)^{i}$ is the $i_{t h}$ voxel of $\operatorname{mask}_{y}(y)$ and $N$ is the number of total voxels. Objective 14 function of B-rmse loss is:

15

$\min _{G}\left(\max _{D} L_{C G A N}(G, D)+\mu L_{1}(G)+\lambda L_{F M}(G, D)+\gamma L_{B-r m s e}(G)\right)$

where $\gamma$ controls the regularization term for the brain area rmse loss.

19 In the ablation study, we found that B-rmse loss contributed to the improvement of the network performance and improved the synthesis accuracy. Notice that B-rmse loss is not the only loss

21 for the generator, there are combination of $L_{1}$ loss, B-rmse loss and feature matching loss for

22 generator. $L_{1}$ loss focuses on the difference of whole output and target and B-rmse loss focuses

23 on the only brain area's difference of output and target.

\section{3D self-attention}

26 Self-attention allows GAN to efficiently model relationships between widely separated spatial

27 regions (Zhang et al., 2018), so that generated images contain realistic details. The image feature

28 map $x \in R^{C \times h * w * d}$ from one intermediate hidden layer of 3D cGAN was transformed into 2 
1 feature spaces $f(x)=W_{f} x$ and $g(x)=W_{g} x$ to calculate the attention. Then, the third feature

2 space $h(x)=W_{h} x$ was used to calculate attention feature map. Since the purpose of utilizing

3 self-attention is to measure the similarity of each voxel with target voxel, we used the similarity

4 scores (attentions) as weights to calculate the weighted sum represent of each target voxel. 3D

5 self-attention module structure is presented in Figure 1.

6

7 Similarity score (attention) was calculated as follow:

$8 \quad \beta_{j, i}=\frac{\exp \left(S_{j, i}\right)}{\sum_{i=1}^{N} \exp \left(S_{j, i}\right)}, \quad$ where $S_{j, i}=f\left(x_{j}\right)^{T} g\left(x_{i}\right)$

9

10 in which, $\beta_{j, i}$ is voxel $j$ 's attention to voxel $i$. We then calculated attention feature for each voxel

$11 j$ by:

12

$13 \quad O_{j}=v\left(\sum_{i=1}^{N} \beta_{j, i} h\left(x_{i}\right)\right)$, where $v(x)=W_{v} x$

14

15 The final output of attention layer is:

16

$17 y_{j}=\alpha O_{j}+x_{j}$

18

19 In the above formulations

20

$21 W_{f} \in R^{\bar{C} \times C}, \quad W_{g} \in R^{\bar{C} \times C}, \quad W_{h} \in R^{\bar{C} \times C}, \quad W_{v} \in R^{C \times \bar{C}}, O \in R^{C \times h * w * d}$

22

$23 W_{f}, W_{g}, W_{h}, W_{v}$ are learned weight matrices by $1 \times 1 \times 13 \mathrm{D}$ convolutions; $C$ is the number of

24 original channels; $\bar{C}$ equals to $C / 8$ for memory efficiency; $h * w * d$ is the number of voxels in

25 one feature map; $\alpha$ is a learnable scalar and it is initialized as 0. 
1 In our network, self-attention is implemented in both generator and discriminator as shown in

2 Figure 2. Generator for conditional GAN is the same as U-net (Ronneberger et al., 2015) structure.

3 When comparing our results with U-net, we added self-attention at both encoder and decoder

4 of U-net to improve the synthesis performance.

5

6 Spectral normalization

7 Spectral normalization is implemented in each layer $g: h_{\text {in }} \rightarrow h_{\text {out }}$ of the neural networks to 8 normalize the weight matrix between two connected layers by controlling the Lipschitz constant.

9 By definition, Lipschitz norm $\|g\|_{\text {Lip }}=\sup _{h} \sigma(\nabla g(h))$, where $\sigma(\cdot)$ is the spectral norm (the 10 largest singular value).

11 Suppose a neural network $f(x, W, a)=W^{L+1} a_{L}\left(W^{L}\left(a_{L-1}\left(W^{L-1}\left(\ldots a_{1}\left(W^{1} x\right) \ldots\right)\right)\right)\right)$, where $12\left\{W^{1}, W^{2}, \ldots, W^{L+1}\right\}$ is the weights set, $\left\{a_{1}, a_{2}, \ldots, a_{L}\right\}$ is the element-wise non-linear 13 activation functions. For the linear layer $g(h)=W h$, the norm is given by:

$\|g\|_{L i p}=\sup _{h} \sigma(\nabla g(h))=\sup _{h} \sigma(\mathrm{W})=\sigma(\mathrm{W})$

17 If the Lipschitz norm of the activation function $\left\|a_{L}\right\|_{L i p}$ is equal to 1 , based on the Cauchy18 Schwarz inequality $\|g 1 \circ g 2\|_{L i p} \leq\|g 1\|_{L i p} \cdot\|g 2\|_{L i p}$, the following bound can be derived:

$$
\|f\|_{L i p} \leq\left\|g_{L+1}\right\|_{L i p} \cdot\left\|a_{L}\right\|_{L i p} \cdot\left\|g_{L}\right\|_{L i p} \cdots\left\|a_{1}\right\|_{L i p} \cdot\left\|g_{1}\right\|_{L i p}=\prod_{l=1}^{L+1}\left\|g_{l}\right\|_{L i p}=\prod_{l=1}^{L+1} \sigma\left(W_{l}\right)
$$

22 The spectral normalization normalizes the spectral norm of the weight matrix $W_{l}$ to get $W_{S N}=$ $23 W_{l} / \sigma\left(W_{l}\right)$. Thus, if $W_{l}$ is normalized as $W_{S N}$, then $\|f\|_{L i p} \leq \prod_{l=1}^{L+1} \sigma\left(W_{S N}\right)=1$ which means $24\|f\|_{\text {Lip }}$ is bounded by 1. Miyato et al (Miyato et al., 2018) have shown the importance of Lipschitz 25 continuity assuring the boundness of statistics. We utilized Spectral normalization in both 26 generator and discriminator of SC-GAN. 
2 In order to prevent overfitting, we added L2 norm regularizations to generator and discriminator,

3 resulting to a final objective function of:

4

$5 \min _{G}\left(\max _{D}\left(L_{C G A N}(G, D)-v_{D} L_{2}(D)\right)+\mu L_{1}(G)+\lambda L_{F M}(G, D)+\gamma L_{B-r m s e}(G)+v_{G} L_{2}(G)\right)$

6

7 where $v_{D}$ and $v_{G}$ control the importance of $L_{2}$ regularization. Since during the training process

8 we minimize the negative discriminator loss for the discriminator training, the above objective

9 function uses $-v_{D} L_{2}(D)$ to regularize discriminator. Note that $L_{2}(D)$ and $L_{2}(G)$ are the

10 constraints on trainable values of discriminator and generator, however, $L_{1}(G)$ is the $L_{1}$ distance

11 between generated output and ground truth.

12 


\section{Experiments}

\section{Study data}

3 Data used in the preparation of this article were obtained from the Alzheimer's Disease

4 Neuroimaging Initiative 3 (ADNI-3) database (http://adni.loni.usc.edu) (Weiner et al., 2017). We

5 downloaded MRI and PET data from ADNI-3 participants. All available from ADNI-3 at the time of

6 this study were used for this study (ADNI-3 is an ongoing project). For PET synthesis task 265

7 images were used (training set $=207$, testing set $=58$ ). For FA and MD synthesis tasks 497 images

8 were used (training set $=398$, testing set $=99)$. For MRI, T1-weighted $(T 1 w)$ and fluid-attenuated

9 inversion recovery (FLAIR) structural magnetic resonance image (MRI) and diffusion-weighted

10 MRI were used. For PET, we used amyloid PET data. For PET synthesis, dataset with complete

11 T1w, FLAIR and amyloid PET sessions with acceptable quality, based on ADNI guidelines were

12 included in the analysis. For diffusion-weighted MRI synthesis, dataset with complete T1w, FLAIR

13 and diffusion-weighted MRI sessions were used (all images were visually inspected).

\section{MRI data collection and preprocessing}

16 MRI imaging of the ADNI-3 was done exclusively on 3T scanners (Siemens, Philips, and GE) using

17 a standardized protocol. 3D T1w with $1 \mathrm{~mm}^{3}$ resolution was acquired using an MPRAGE sequence

18 (on Siemens and Philips scanners) and FSPGR (on GE scanners). For FLAIR images, a 3D sequence

19 with similar resolution as T1w images was acquired, which provided the opportunity for accurate

20 intrasubject intermodal co-registration. MPRAGE T1w MRI scans were acquired using the

21 following parameters: $\mathrm{TR}=2300 \mathrm{~ms}, \mathrm{TE}=2.98 \mathrm{~ms}$, FOV $=240 \times 256 \mathrm{~mm}^{2}$, matrix $=240 \times 256$

22 (variable slice number), $\mathrm{Tl}=900 \mathrm{~ms}$, flip angle $=9$, effective voxel resolution $=1 \times 1 \times 1 \mathrm{~mm}^{3}$. The

23 FSPGR sequence was acquired using sagittal slices, TR $=7.3 \mathrm{~ms}$, TE $=3.01 \mathrm{~ms}, \mathrm{FOV}=256 \times 256$

$24 \mathrm{~mm}^{2}$, matrix $=256 \times 256$ (variable slice number), $\mathrm{TI}=400 \mathrm{~ms}$, flip angle $=11$, effective voxel

25 resolution $=1 \times 1 \times 1 \mathrm{~mm}^{3}$. 3D FLAIR images were acquired using sagittal slices, TR $=4,800 \mathrm{~ms}$,

$26 \mathrm{TE}=441 \mathrm{~ms}, \mathrm{FOV}=256 \times 256 \mathrm{~mm}^{2}$, matrix $=256 \times 256$ (variable slice number), $\mathrm{Tl}=1650 \mathrm{~ms}$, flip

27 angle $=120$, effective voxel resolution $=1 \times 1 \times 1.2 \mathrm{~mm}^{3}$. 
1 T1w preprocessing and parcellation was done using the FreeSurfer (v5.3.0) software package,

2 which is freely available (Fischl, 2012), and data processing using the Laboratory of Neuro Imaging

3 (LONI) pipeline system (http://pipeline.loni.usc.edu) (Dinov et al., 2010, 2009; Moon et al., 2015;

4 Torri et al., 2012), similar to (Sepehrband et al., 2018; Sta Cruz et al., 2019). Field corrected,

5 intensity normalized images were filtered using non-local mean filtering to reduce the noise, and

6 the outputs were used for the analysis. FLAIR images of each individuals were corrected for non-

7 uniform field inhomogeneity using N4ITK module (Tustison et al., 2010) of Advanced

8 Normalization Tools (ANTs) (Avants et al., 2009). FLAIR images were then co-registered to T1w

9 images using antsIntermodalityIntrasubject ANTs module.

11 Diffusion MRI is a quantitative modality and contain microstructural information about brain

12 tissue (Le Bihan et al., 2001; Sepehrband et al., 2017, 2015). Therefore, it was used as a 13 challenging synthesis target from T1 and FLAIR, which are mainly qualitative maps. Diffusion MRI

14 data was acquired using the following parameters: 2D echo-planar axial imaging, with sliced 15 thickness of $2 \mathrm{~mm}$, in-plane resolution of $2 \mathrm{~mm}^{2}$ (matrix size of $1044 \times 1044$ ), flip angle of $90^{\circ}, 48$

16 diffusion-weighted images with 48 uniformly distributed diffusion-encodings with $b$-value=1000 $17 \mathrm{~s} / \mathrm{mm}^{2}$ and 7 non-diffusion-weighted images. Diffusion MRI preprocessing and diffusion tensor

18 imaging (DTI) fitting were performed were as described in (Sepehrband et al., 2019b, 2019a). In 19 brief, images were corrected for eddy current distortion and for involuntary movement, using 20 FSL TOPUP and EDDY tools (Andersson et al., 2012, 2003). DTI was then fitted to diffusion data 21 using Quantitative Imaging Toolkit (Cabeen et al., 2018). Fractional anisotropy (FA) and mean 22 diffusivity (MD) maps were used for the synthesis task.

\section{PET data collection and preprocessing}

25 Amyloid PET analysis was performed according to UC Berkeley PET methodology for quantitative measurement (Baker et al., 2017; Landau et al., 2015, 2014; Schöll et al., 2016). Participants were

27 imaged by Florbetapir ( ${ }^{18} \mathrm{~F}-\mathrm{AV}-45$, Avid), or ${ }^{18}$ F-Florbetaben (NeuraCeq, Piramal). Six five-minute 28 frames of PET images were acquired 30 to 60 minutes post injection. Each extracted frame is co29 registered to the first extracted frame and then combined into one image, which lessens the 
1 subject motion artifacts. The combined image had the same image resolution of the original PET

2 image (2mm isotropic voxels). All PET images were co-registered on T1w MRI. Quantitative

3 measurement was done based on Standard Uptake Value ratio (SUVR). The brain mask was,

4 obtained from T1w analysis was applied on co-registered. T1w, FLAIR and PET images. Examples

5 of a set of input and target images are presented in Figure 3.

Implementation, baseline models

8 In order to rigorously assess the performance of the SC-GAN, we have compared it with current

9 well-developed medical image synthesis networks, including: 2D cGAN, 3D cGAN and Attention 10 cGAN (Att cGAN). 2D cGAN was adopted from Ouyang et al (Ouyang et al., 2019), which proposed

11 it for PET synthesis task. 3D cGAN was firstly proposed by Wang et al (Wang et al., 2019) for PET 12 image synthesis from low dose PET images. Attention cGAN was designed based on the attention 13 module proposed by Oktay et al (Oktay et al., 2018), which incorporated the 3D attention module 14 in the U-net architecture for the application of pancreas segmentation (assisted by image 15 synthesis task). The same 3D attention module was also adopted by Liu et al(Liu, 2019) in Cycle16 GAN medical image synthesis network. For a fair comparison, we incorporated aforementioned 17 3D attention module in conditional GAN, here referred to as Att-GAN, and compared it with SCGAN. Note that the self-attention module has a different mechanism compared with attention module. Unlike the attention module, the self-attention exploits the dependencies of each pair of positions in the same feature map to get attention matrix, then use attention matrix to

21 reconstruct representation and combine it with the same input data. All 3 baseline models and

22 SC-GAN were implemented using TensorFlow (1.12.2) and deployed training on an NVIDIA GPU 23 cluster equipped with eight V100 GPUs (Cisco UCS C480 ML). All four sets of results are used to 24 analyze and compare different networks' performance.

\section{Image preparation prior to training}

27 For the PET synthesis task, 207 pairs of T1W and FLAIR images were used as training data and 58 28 pairs of T1w and FLIAR images were used as test data. For the DTI-MD and DTI-FA synthesis tasks, 
1 images were used as test data. PET and DTI were upsampled to have the same resolution as the

2 T1 and FLAIR, i.e. $256 \times 256 \times 256$. We implemented Z-score normalization for all four tasks, then

3 applied min-max rescaling to scale the voxels' intensity between 0 to 1 prior to the training.

4 Synthesis methods could be improved with intensity normalization, but are robust to the choice

5 of the normalization (Reinhold et al., 2019).

$7 \quad$ Training and testing

8 2D cGAN was implemented similar to (Ouyang et al., 2019). We utilized pix2pix structure (U-net 9 generator and patch GAN discriminator) with feature matching loss and regularization. 3D cGAN 10 was implemented similar to (Wang et al., 2019) and Att cGAN was implemented similar to (Liu, 11 2019; Oktay et al., 2018). We performed 5-fold cross validation during the hyperparameter 12 tuning phase for all four networks to get the optimum hyperparameters.

13 SC-GAN network architecture is illustrated in Figure 2 and the loss function formulation was 14 described in equation 14. The optimum result was obtained with the following hyperparameters: $15 \mu=200, \gamma=200, \lambda=20, v_{G}=0.001, v_{D}=0.001$, batch size $=1$. Learning rate starts as 0.001 16 and cosine decay was used to continuously shrink the learning rate during the training process.

\section{Evaluation criteria}

19 Three image quality metrics were used to evaluate the performance of the synthesis task: normalized root mean square error (NRMSE), peak signal-to-noise ratio (PSNR) and structural

21 similarity (SSIM). NRMSE reflects the normalized error without being affected by the range of the

22 voxel values. Thus, NRMSE could be used to compare the performances of the network on 23 different tasks directly. To enable a direct comparison between 2D cGAN and 3D networks, we 24 evaluated the 3D output of the 2D network directly.

26 Ablation study

27 In order to analyze the contribution of each component of SC-GAN, we performed an ablation study. Five ablation tests were conducted for the proposed network, namely: SC-GAN 1) without 
1 self-attention module, 2) without adversarial learning, 3) without brain area rmse loss, 4) without

2 spectral normalization, and 5) without feature matching loss.

3

$4 \quad$ Evaluating synthesized PET

5 A secondary analysis was performed to compare SC-GAN results against ground truth PET.

6 Amyloid-b $(A \beta)$ uptake were estimated from PET and synthesized PET. The $A \beta$ uptake values were

7 then compared across clinically relevant regions. While the focus of the study was on proposing

8 and optimizing a neuroimage synthesis technique, this evaluation was performed to examine

9 whether the PET synthetization from MRI can substitute the PET imaging. Standard uptake value

10 ratio (SUVR) of the $A \beta$ were calculated across subcortical and cortical regions of 10 randomly

11 selected individuals from ADNI-3 cohort. SUVR values of 110 regions per participants were

12 compared between PET and synthesized PET. SUVRs across these regions of interest were derived

13 using the Desikan-Killiany atlas, which were parcellated on T1w images using FreeSurfer pipeline,

14 as explained in B. MRI data collection and preprocessing section. PET images that were used for

15 training were normalized using min-max normalization approach. Therefore, test PET images

16 were also normalized using the same approach before comparison. 


\section{Results}

2 The learning curves of the GANs that were used for PET, FA and MD synthesis tasks are presented

3 in the Figure 4. Learning curves demonstrate network performance across training epochs.

4 Average performance of applying the trained network on the test data is presented in Figure 5,

5 and the qualitative assessments are presented in Figures 6-8.

Quantitative assessment

8 The learning curves show that all networks were successfully optimized, reaching the plateau

9 within the range of the study epochs (Figure 4). 3D cGAN and SC-GAN networks showed a smooth

10 and stable pattern in their optimization curve, while 2D cGAN and att GAN demonstrated a

11 degree of fluctuation during the learning. The pattern of the learning curves across tasks was

12 similar in SSIM and NRMSE. However, the PSNR was slightly different across tasks, with PET tasks

13 resulted to the highest PSNR (Figure 4).

15 Regardless of the evaluation metric or the synthesis task, SC-GAN outperformed other networks, 16 resulting to the lowest NRMSE and the highest PSNR and SSIM (Figure 5 and Table 1). The NRMSE 17 results showed that error of SC-GAN was $18 \%, 24 \%$ and $29 \%$ lower compared to 2 D cGAN across

18 FA, PET and MD tasks, respectively. Across all tasks, the 2D network resulted to the lowest 19 performance.

21 All 3D networks outperformed the 2D network, highlighting the importance of incorporating 3D

22 information into deep learning networks. SC-GAN outperformed 3D cGAN and att GAN in all three

23 tasks across all evaluation metrics. The increased performance of the SC-GAN was more evident

24 in the PET task, followed by FA and MD tasks.

26 The ablation test showed that the major contributors to SC-GAN performance are the adversarial

27 learning and the self-attention module, followed by B-rmse and spectral normalization modules

28 (Figure 9 and Table 2). Spectral normalization contributed to the stabilization of the SC-GAN 
1 training and feature matching loss contributed to generate synthesis result with more natural

2 statistics at multiples scales.

3

4

5

6

7 detail and less amount of artifact.

\section{Qualitative assessment} dimensions.

Figure 6-8 compare the studied networks qualitatively. To assess the quality of the synthesis images in 3D, images were view across different plans: Axial images for PET synthesis (Figure 6), coronal images for FA synthesis (Figure 7) and sagittal images for MD (Figure 8). Because 2D cGAN was trained on the sagittal images, the sagittal view of the synthesized result provided the best result for the 2D network (e.g. MD task: Figure 8), while the axial and coronal views presented visual discontinuity and distortion (e.g. PET and FA tasks: Figure 6 and 7). Even at sagittal view, 2D GAN generated sharp artificial boundaries (ventricle boundaries in Figure 8). 3D network did not suffer from either of these shortcomings, presenting stable results across image

SC-GAN results were visually closest to the ground truth data in comparison with other networks. In particular, SC-GAN was able to capture certain details in the image that were hidden to other networks. For example, structural boundaries at brain stem of the FA images were captured by SC-GAN (green dotted circle in Figure 7), but these details were smoothed out when other networks were used. Cingulum bundle (blue arrows, Figure 7) and superficial white matter (red arrow, Figure 7) were not generated with 3D cGAN and 2D cGAN, respectively. These details were successfully generated by SC-GAN. We also noted that att GAN failed to capture high intensity FA across the white matter (yellow arrows, Figure 7), whereas SC-GAN demonstrated a similar intensity profile as the ground truth. It should be noted that the SC-GAN also did not generated an exact match of the ground truth - artificial and incorrect features were observed. Results from MD synthesis (Figure 8) also showed that SC-GAN resulted to the generation of a map that is closest to the ground truth in comparison with other networks and contains higher degree of 
Here we presented an efficient end-to-end framework for multi-modal 3D medical image synthesis (SC-GAN) and validated it in PET, FA and MD synthesis applications. In order to design and optimize the network, we added a 3D self-attention module to the conditional GAN, which models the similarity between adjacent and widely separated voxels of the 3D image. We also employed spectral normalization and feature matching to stabilize the training process and ensure SC-GAN generate more realistic details (less artifacts). SC-GAN technique also allows multi-modal (multi-channel) 3D image input. We showed that SC-GAN significantly outperformed the state-of-the-art techniques, enabling reliable and robust deep learning-based medical image synthesis. SC-GAN is made available via https://github.com/Haoyulance/SC-GAN.

To obtain a generalized multi-modal 3D neuroimaging synthesis technique, SC-GAN incorporates

14 adversarial learning, self-attention mechanism and stable learning strategy. SC-GAN network

15 structure is demonstrated in Figure 2. The generator and discriminator are equipped with 3D self-

16 attention modules, which can capture both short-and-long range dependencies for each feature

17 vector during the learning process.

The self-attention feature makes SC-GAN a context-aware network, providing additional degree of freedom to the synthesis process. The ablation experiment conducted in this study showed that self-attention module contributed the most to the improvement of the conventional 3D GAN. Previous studies have shown that self-attention can be effective in other medical image analysis applications as well. Zhao et al (Zhao et al., 2020) combined object recognition network and self-attention guided GAN into one training process to handle tumor detection task. Li et al

25 (Li et al., 2020) incorporated self-attention and auto encoder perceptual loss into convolutional neural network to denoise low dose CT.

While adding attention module improved the 3D cGAN, it provided less accurate results in comparison with SC-GAN that uses self-attention module. Att cGAN employs attention gate that 
1 upsampling phase. Since training process of Att cGAN is also guided by the attention gate module, network performance was better than 3D cGAN. Qualitative results also showed that Att cGAN can generated better results compared to 3D cGAN.

3D Medical image processing tasks often face dimensionality challenges, and GAN is no exception (Lundervold and Lundervold, 2019). 3D cGAN resulted to oversmoothed images in FA synthesis task and generated a large amount of striping artifact that resulted to blurring of the edges at

9 techniques, namely feature matching loss, spectral normalization loss, L1 loss and brain area RMSE loss, allowing stable training on high dimensional input data (e.g. the input image size of $\mathrm{N}$

$11 \times 256 \times 256 \times 256 \times 2$ was used in this study).

13 The SC-GAN without adversarial learning resulted to a lower synthesis accuracy compared to the 14 main implementation. SC-GAN without adversarial learning abandons the discriminator during 15 the training phase. Since generator is a U-net like encoder-decoder (Çiçek et al., 2016;

16 Ronneberger et al., 2015), the SC-GAN without adversarial learning is technically a 3D U-net with

17 3D self-attention module. The synthesis results of SC-GAN with and without the adversarial 18 learning showed that the adversarial learning empowers the training process and could extend 19 the plateau of the learning curve.

21 We incorporated a feature matching loss as part of the generator loss to stabilizes the training 22 by forcing the generator to produce natural statistics at multiples scales. The discriminator takes

23 target and synthesis images as inputs sequentially (Figure 2). Then, the cross-entropy loss is 24 calculated to update the weights using a back-propagation approach. The feature matching loss 25 uses the feature maps that are generated in the discriminator phase to produce similar output 26 to the target image by minimizing the error associated with image spatial features.

28 The spectral normalization was used to stabilize the training process and prevent training from collapsing. Spectral normalization utilizes the Lipschitz continuity concept to impose constraint 
1 on the solution space (Hager, 1979) which stabilized SC-GAN training process (Miyato et al., 2018).

2 Spectral normalization uses the Cauchy-Schwarz inequality on the Lipschitz continuity to bound

3 the solution space, which stabilize the optimization.

Several recent works have used adversarial learning strategy for medical image synthesis (Li et al., 2020; Liu, 2019; Lundervold and Lundervold, 2019; Ouyang et al., 2019; Wang et al., 2019; Zhao et al., 2020). Most of the medical image synthesis and reconstruction works have been implemented using 2D or 2.5D input images (Li et al., 2020; Ouyang et al., 2019; Zhao et al., 2020). One drawback of 2D GAN is that the network can only utilizes one 2D image of axial, coronal or sagittal each time, and therefore, the synthesis 3D images present visual discontinuity, which appears similar to stripping artifact (Figure 7 and 8). To evaluate the benefits of 3D implementation, we compared the performances of 2D cGAN and 3D networks. We observed intensity discontinuity and distortion in the synthesis results of the 2D cGAN, which highlights

Recent works have shown that 3D GAN can be utilized to improve the accuracy of the medical

17 imaging synthesis (Liu, 2019; Wang et al., 2019). To the best of our knowledge Wang et al first expanded the medical image synthesis GAN from 2D to 3D by utilizing 3D convolution and transposed convolution to achieve high-quality PET image estimation from low dose PET images (Wang et al., 2019). In order to rigorously assess SC-GAN, two existing 3D synthesis methods (3D cGAN and Att cGAN) were compared with SC-GAN. SC-GAN resulted to the highest performance

22 and most stable learning curves (Figures 4-5).

It should be noted that while neuroimaging synthesis has drastically improved over the past five years, we do not think that synthesis can entirely substitute a given modality that is different in nature (for example PET). To assess the performance of image synthesis for detecting pathology

27 in a cross-modal application, we estimated regional Amyloid update from synthesis PET and 28 compared it with the ground truth PET (Figure 10). We noted a significant correlation between 29 PET and synthesis PET across subcortical and cortical regions ( $p<0.0001$ across all ten tested 
1 participants). Results were consistent across all test subjects, with correlation coefficient ranging

2 from $r=0.67$ to $r=0.95$ (all with $p<0.0001$ ). While synthesis PET SUVR values were significantly

3 correlated with those from ground truth PET, we observed that the error rate is higher when

4 SUVR of the PET images are higher. These SUVR range corresponds to regions with high clinical

5 value, reflecting neurodegenerative pathology (high $A \beta$ uptake). Therefore, our results suggest

6 that synthesis PET cannot substitute PET imaging, because pathological and clinically relevant

7 molecular information in PET may not be detected by synthesizing PET that is obtained from MRI

8 (which are mainly contain structural information). Nevertheless, this limitation does not damper

9 the significance of medical image synthesis but calls for a careful design/application when image

10 synthesis is used. For example, studies have shown that incorporating low-dose PET as synthesis

11 input, reliable transformation can be achieved (Chen et al., 2019; Ouyang et al., 2019; Wang et

12 al., 2019) .

14 The focus of this work was on enabling multi-modal 3D neuroimage synthetization with GAN. The 15 proposed method for this multi-modal 3D neuroimage synthesis (SC-GAN) was evaluated on the 16 challenging task of PET and DTI synthesis to aid rigorous optimization of the network. SC-GAN is 17 not intended to substitute PET with MRI-based PET synthesis. SC-GAN was designed and assessed 18 to enable robust and stable multi-modal 3D neuroimaging synthesis. Future work will explore SC19 GAN application. For example, SC-GAN may be used to combine MRI with low-dose PET to 20 improve the efficacy of the existing techniques (Chen et al., 2019; Ouyang et al., 2019). We also

21 expect that neuroimaging techniques with high number of repetitions such as functional and 22 diffusion MRI (Ning et al., 2018) may benefit from SC-GAN, which is a future direction of our work. 


\section{Acknowledgement}

2 This work was supported by NIH grants: 2P41EB015922-21, 1P01AG052350-01, U54EB020406,

3 USC ADRC 5P50AG005142. The content is solely the responsibility of the authors and does not 4 necessarily represent the official views of the NIH.

5 ADNI: Data collection and sharing for this project was funded by the Alzheimer's Disease 6 Neuroimaging Initiative (ADNI) (National Institutes of Health Grant U01 AG024904) and DOD 7 ADNI (Department of Defense award number W81XWH-12-2-0012). ADNI is funded by the 8 National Institute on Aging, the National Institute of Biomedical Imaging and Bioengineering, and 9 through generous contributions from the following: AbbVie, Alzheimer's Association; Alzheimer's 10 Drug Discovery Foundation; Araclon Biotech; BioClinica, Inc.; Biogen; Bristol-Myers Squibb 11 Company; CereSpir, Inc.; Cogstate; Eisai Inc.; Elan Pharmaceuticals, Inc.; Eli Lilly and Company; 12 Eurolmmun; F. Hoffmann-La Roche Ltd and its affiliated company Genentech, Inc.; Fujirebio; GE

13 Healthcare; IXICO Ltd.; Janssen Alzheimer Immunotherapy Research \& Development, LLC.; 14 Johnson \& Johnson Pharmaceutical Research \& Development LLC.; Lumosity; Lundbeck; Merck \&

15 Co., Inc.; Meso Scale Diagnostics, LLC.; NeuroRx Research; Neurotrack Technologies; Novartis 16 Pharmaceuticals Corporation; Pfizer Inc.; Piramal Imaging; Servier; Takeda Pharmaceutical 17 Company; and Transition Therapeutics. The Canadian Institutes of Health Research is providing 18 funds to support ADNI clinical sites in Canada. Private sector contributions are facilitated by the 19 Foundation for the National Institutes of Health (www.fnih.org). The grantee organization is the 20 Northern California Institute for Research and Education, and the study is coordinated by the 21 Alzheimer's Therapeutic Research Institute at the University of Southern California. ADNI data 22 are disseminated by the Laboratory for Neuro Imaging at the University of Southern California. 
Andersson, J.L.R., Skare, S., Ashburner, J., 2003. How to correct susceptibility distortions in spinecho echo-planar images: Application to diffusion tensor imaging. Neuroimage 20, 870888. https://doi.org/10.1016/S1053-8119(03)00336-7

Andersson, J.L.R., Xu, J., Yacoub, E., Auerbach, E., Moeller, S., Ugurbil, K., 2012. A Comprehensive Gaussian Process Framework for Correcting Distortions and Movements in Diffusion Images, in: Joint Annual Meeting ISMRM-ESMRMB. p. 2426.

Avants, B.B., Tustison, N., Song, G., 2009. Advanced Normalization Tools (ANTS). Insight J. 1-35. Baker, S.L., Lockhart, S.N., Price, J.C., He, M., Huesman, R.H., Schonhaut, D., Faria, J., Rabinovici, G., Jagust, W.J., 2017. Reference tissue-based kinetic evaluation of 18F-AV-1451 for tau imaging. J. Nucl. Med. 58, 332-338.

Cabeen, R.P., Laidlaw, D.H., Toga, A.W., 2018. Quantitative Imaging Toolkit : Software for Interactive 3D Visualization, Data Exploration, and Computational Analysis of Neuroimaging Datasets, in: ISMRM-ESMRMB Abstracts. pp. 12-14.

Chen, K.T., Gong, E., Bezerra, F., Macruz, D.C., Xu, J., 2019. Ultra - Low-Dose 18 F-Florbetaben Amyloid PET Imaging Using Deep Learning with Multi-Contrast MRI Inputs.

Çiçek, Ö., Abdulkadir, A., Lienkamp, S.S., Brox, T., Ronneberger, O., 2016. 3D U-Net: learning dense volumetric segmentation from sparse annotation, in: International Conference on Medical Image Computing and Computer-Assisted Intervention. Springer, pp. 424-432.

Dinov, I., Lozev, K., Petrosyan, P., Liu, Z., Eggert, P., 2010. Neuroimaging Study Designs, Computational Analyses and Data Provenance Using the LONI Pipeline. PLoS One 5, e13070. https://doi.org/10.1371/journal.pone.0013070

Dinov, I.D., Van Horn, J.D., Lozev, K.M., Magsipoc, R., Petrosyan, P., Liu, Z., MacKenzie-Graham, A., Eggert, P., Parker, D.S., Toga, A.W., 2009. Efficient, distributed and interactive neuroimaging data analysis using the LONI pipeline. Front. Neuroinform. 3.

Fischl, B., 2012. FreeSurfer. Neuroimage 62, 774-781. https://doi.org/https://doi.org/10.1016/j.neuroimage.2012.01.021

Goodfellow, I.J., Pouget-Abadie, J., Mirza, M., Xu, B., Warde-Farley, D., Ozair, S., Courville, A., Bengio, Y., 2014. Generative adversarial nets. Adv. Neural Inf. Process. Syst. 3, 2672-2680. https://doi.org/10.3156/jsoft.29.5_177_2

Hager, W.W., 1979. LIPSCHITZ-CONTINUITY FOR CONSTRAINED PROCESSES. SIAM J. Control Optim. 17, 321-338.

Hiasa, Y., Otake, Y., Takao, M., Matsuoka, T., Takashima, K., Carass, A., Prince, J.L., Sugano, N., Sato, Y., 2018. Cross-modality image synthesis from unpaired data using CycleGAN, in: International Workshop on Simulation and Synthesis in Medical Imaging. Springer, pp. 3141.

Huang, H., Yu, P.S., Wang, C., 2018. An Introduction to Image Synthesis with Generative Adversarial Nets 1-17.

Isola, P., Zhu, J.Y., Zhou, T., Efros, A.A., 2017. Image-to-image translation with conditional adversarial networks, in: Proceedings - 30th IEEE Conference on Computer Vision and Pattern Recognition, CVPR 2017. pp. 5967-5976. https://doi.org/10.1109/CVPR.2017.632

Landau, S.M., Fero, A., Baker, S.L., Koeppe, R., Mintun, M., Chen, K., Reiman, E.M., Jagust, W.J., 2015. Measurement of longitudinal $\beta$-amyloid change with $18 \mathrm{~F}$-florbetapir PET and 
standardized uptake value ratios. J. Nucl. Med. 56, 567-574.

Landau, S.M., Thomas, B.A., Thurfjell, L., Schmidt, M., Margolin, R., Mintun, M., Pontecorvo, M., Baker, S.L., Jagust, W.J., Initiative, A.D.N., 2014. Amyloid PET imaging in Alzheimer's disease: a comparison of three radiotracers. Eur. J. Nucl. Med. Mol. Imaging 41, 13981407.

Le Bihan, D., Mangin, J.F., Poupon, C., Clark, C.A., Pappata, S., Molko, N., Chabriat, H., 2001. Diffusion tensor imaging: Concepts and applications. J. Magn. Reson. Imaging 13, 534-546. https://doi.org/10.1002/jmri.1076

Li, M., Hsu, W., Xie, X., Cong, J., Gao, W., 2020. SACNN: Self-Attention Convolutional Neural Network for Low-Dose CT Denoising with Self-supervised Perceptual Loss Network. IEEE Trans. Med. Imaging XX, 1-1. https://doi.org/10.1109/tmi.2020.2968472

Liu, X., 2019. Unpaired Data based Cross-domain Synthesis and Segmentation Using Attention Neural Network 987-1000.

Lundervold, A.S., Lundervold, A., 2019. An overview of deep learning in medical imaging focusing on MRI. Z. Med. Phys. 29, 102-127. https://doi.org/10.1016/j.zemedi.2018.11.002

Mirza, M., Osindero, S., 2014. Conditional Generative Adversarial Nets 1-7.

Miyato, T., Kataoka, T., Koyama, M., Yoshida, Y., 2018. Spectral normalization for generative adversarial networks. 6th Int. Conf. Learn. Represent. ICLR 2018 - Conf. Track Proc.

Moon, S.W., Dinov, I.D., Kim, J., Zamanyan, A., Hobel, S., Thompson, P.M., Toga, A.W., 2015. Structural neuroimaging genetics interactions in Alzheimer's disease. J. Alzheimer's Dis. 48, 1051-1063. https://doi.org/10.3233/JAD-150335

Nash, J.F., 1950. Equilibrium points in n-person games. Proc. Natl. Acad. Sci. 36, 48-49.

Nie, D., Trullo, R., Lian, J., Petitjean, C., Ruan, S., Wang, Q., Shen, D., 2017. Medical image synthesis with context-aware generative adversarial networks, in: International Conference on Medical Image Computing and Computer-Assisted Intervention. Springer, pp. 417-425.

Nie, D., Trullo, R., Lian, J., Wang, L., Petitjean, C., Ruan, S., Wang, Q., Shen, D., 2018. Medical Image Synthesis with Deep Convolutional Adversarial Networks. IEEE Trans. Biomed. Eng. 65, 2720-2730. https://doi.org/10.1109/TBME.2018.2814538

Ning, L., Bonet-Carne, E., Grussu, F., Sepehrband, F., Kaden, E., Veraart, J., Blumberg, S.B., Khoo, C.S., Palombo, M., Coll-Font, J., Scherrer, B., Warfield, S.K., Karayumak, S., Rathi, Y., Koppers, S., Weninger, L., Ebert, J., Merhof, D., Moyer, D., Pietsch, M., Christianens, D., Teixeira, R., Tournier, J.-D., Zhylka, A., Pluim, J., Rudrapatna, U., Evans, J., Charron, C., Jones, D.K., Tax, C., 2018. Muti-shell diffusion MRI harmonisation and enhancement challenge (MUSHAC): progress and results, in: Medical Image Computing and Computer Assisted Intervention (MICCAI). Granada, Spain.

Oktay, O., Schlemper, J., Folgoc, L. Le, Lee, M., Heinrich, M., Misawa, K., Mori, K., Mcdonagh, S., Hammerla, N.Y., Kainz, B., Glocker, B., Rueckert, D., 2018. Attention U-Net : Learning Where to Look for the Pancreas.

Ouyang, J., Chen, K.T., Gong, E., Pauly, J., Zaharchuk, G., 2019. Ultra-low-dose PET reconstruction using generative adversarial network with feature matching and taskspecific perceptual loss. Med. Phys. 46, 3555-3564. https://doi.org/10.1002/mp.13626

Reinhold, J.C., Dewey, B.E., Carass, A., Prince, J.L., 2019. Evaluating the impact of intensity 
normalization on MR image synthesis 126. https://doi.org/10.1117/12.2513089

Ronneberger, O., Fischer, P., Brox, T., 2015. U-net: Convolutional networks for biomedical image segmentation, in: Lecture Notes in Computer Science (Including Subseries Lecture Notes in Artificial Intelligence and Lecture Notes in Bioinformatics). Springer, pp. 234-241. https://doi.org/10.1007/978-3-319-24574-4_28

Roy, S., Carass, A., Jog, A., Prince, J.L., Lee, J., 2014. MR to CT registration of brains using image synthesis, in: Medical Imaging 2014: Image Processing. International Society for Optics and Photonics, p. 903419.

Schöll, M., Lockhart, S.N., Schonhaut, D.R., O’Neil, J.P., Janabi, M., Ossenkoppele, R., Baker, S.L., Vogel, J.W., Faria, J., Schwimmer, H.D., 2016. PET imaging of tau deposition in the aging human brain. Neuron 89, 971-982.

Sepehrband, F., Cabeen, R.P., Barisano, G., Sheikh-Bahaei, N., Choupan, J., Law, M., Toga, A.W., 2019a. Nonparenchymal fluid is the source of increased mean diffusivity in preclinical Alzheimer's disease. Alzheimer's Dement. Diagnosis, Assess. Dis. Monit. 11, 348-354. https://doi.org/10.1016/j.dadm.2019.03.002

Sepehrband, F., Cabeen, R.P., Choupan, J., Barisano, G., Law, M., Toga, A.W., 2019b. Perivascular space fluid contributes to diffusion tensor imaging changes in white matter. Neuroimage 197, 243-254. https://doi.org/10.1016/j.neuroimage.2019.04.070 Sepehrband, F., Clark, K.A., Ullmann, J.F.P., Kurniawan, N.D., Leanage, G., Reutens, D.C., Yang, Z., 2015. Brain tissue compartment density estimated using diffusion-weighted MRI yields tissue parameters consistent with histology. Hum. Brain Mapp. 36, 3687-3702. https://doi.org/10.1002/hbm.22872

Sepehrband, F., Lynch, K.M., Cabeen, R.P., Gonzalez-Zacarias, C., Zhao, L., D’Arcy, M., Kesselman, C., Herting, M.M., Dinov, I.D., Toga, A.W., Clark, K.A., 2018. Neuroanatomical morphometric characterization of sex differences in youth using statistical learning. Neuroimage 172, 217-227. https://doi.org/10.1016/j.neuroimage.2018.01.065

Sepehrband, F., O'Brien, K., Barth, M., 2017. A time-efficient acquisition protocol for multipurpose diffusion-weighted microstructural imaging at 7 Tesla. Magn. Reson. Med. 78, 2170-2184. https://doi.org/10.1002/mrm.26608

Shin, H.-C., Tenenholtz, N.A., Rogers, J.K., Schwarz, C.G., Senjem, M.L., Gunter, J.L., Andriole, K.P., Michalski, M., 2018. Medical image synthesis for data augmentation and anonymization using generative adversarial networks, in: International Workshop on Simulation and Synthesis in Medical Imaging. Springer, pp. 1-11.

Sta Cruz, S., Dinov, I.D., Herting, M.M., Gonzalez-Zacarias, C., Kim, H., Toga, A.W., Sepehrband, F., 2019. Imputation Strategy for Reliable Regional MRI Morphological Measurements. Neuroinformatics. https://doi.org/10.1007/s12021-019-09426-x

Torri, F., Dinov, I.D., Zamanyan, A., Hobel, S., Genco, A., Petrosyan, P., Clark, A.P., Liu, Z., Eggert, P., Pierce, J., Knowles, J.A., Ames, J., Kesselman, C., Toga, A.W., Potkin, S.G., Vawter, M.P., Macciardi, F., 2012. Next generation sequence analysis and computational genomics using graphical pipeline workflows. Genes (Basel). 3, 545-575. https://doi.org/10.3390/genes3030545

Tustison, N.J., Avants, B.B., Cook, P.A., Zheng, Y., Egan, A., Yushkevich, P.A., Gee, J.C., 2010. N4ITK: improved N3 bias correction. IEEE Trans. Med. Imaging 29, 1310-1320.

Wang, T.C., Liu, M.Y., Zhu, J.Y., Tao, A., Kautz, J., Catanzaro, B., 2018. High-Resolution Image 
Synthesis and Semantic Manipulation with Conditional GANs, in: Proceedings of the IEEE Computer Society Conference on Computer Vision and Pattern Recognition. pp. 87988807. https://doi.org/10.1109/CVPR.2018.00917

Wang, Y., Biting, Y., Lei Wangc, Chen Zud, David S. Lalushe, Weili Linf, Xi Wug, Jiliu Zhoua, g, Dinggang Shenf, $h{ }^{*}$, and Luping Zhoub, $c, *, 2019$. 3D conditional generative adversarial networks for high-quality PET image estimation at low dose. Physiol. Behav. 176, 139-148. https://doi.org/10.1016/j.physbeh.2017.03.040

Weiner, M.W., Veitch, D.P., Aisen, P.S., Beckett, L.A., Cairns, N.J., Green, R.C., Harvey, D., Jack, C.R., Jagust, W., Morris, J.C., Petersen, R.C., Salazar, J., Saykin, A.J., Shaw, L.M., Toga, A.W., Trojanowski, J.Q., 2017. The Alzheimer's Disease Neuroimaging Initiative 3: Continued innovation for clinical trial improvement. Alzheimer's Dement. 13, 561-571. https://doi.org/10.1016/j.jalz.2016.10.006

Yi, X., Walia, E., Babyn, P., 2019. Generative adversarial network in medical imaging: A review. Med. Image Anal. 101552.

Zhang, H., Goodfellow, I., Metaxas, D., Odena, A., 2018. Self-attention generative adversarial networks. arXiv Prepr. arXiv1805.08318.

Zhao, J., Li, D., Kassam, Z., Howey, J., Chong, J., Chen, B., 2020. Tripartite-GAN : Synthesizing liver contrast-enhanced MRI to improve tumor detection. Med. Image Anal. 63, 101667. https://doi.org/10.1016/j.media.2020.101667

Zhu, J.Y., Park, T., Isola, P., Efros, A.A., 2017. Unpaired Image-to-Image Translation Using CycleConsistent Adversarial Networks. Proc. IEEE Int. Conf. Comput. Vis. 2017-Octob, 22422251. https://doi.org/10.1109/ICCV.2017.244 

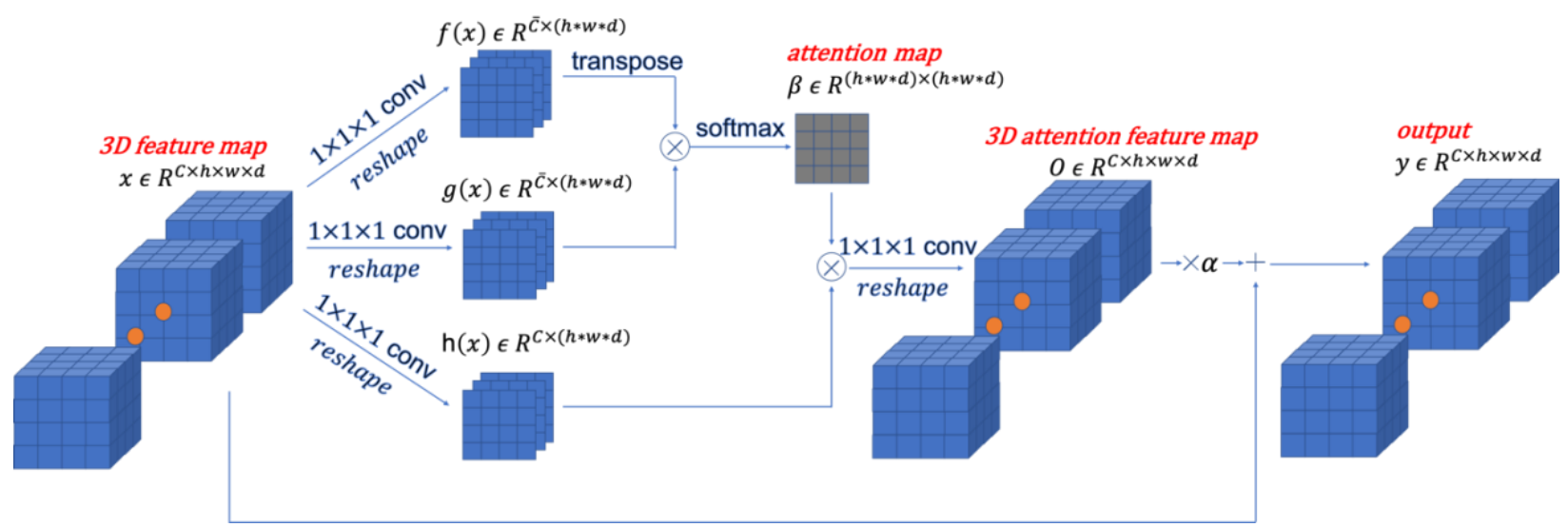

Figure 1. 3D self-attention module. This Figure is a schematic view of the self-attention module of SC-GAN. The first layer represents the input data. Attention map exploit the similarity of each pair of convolved images and combine it with the input data to generate the output of the selfattention module.

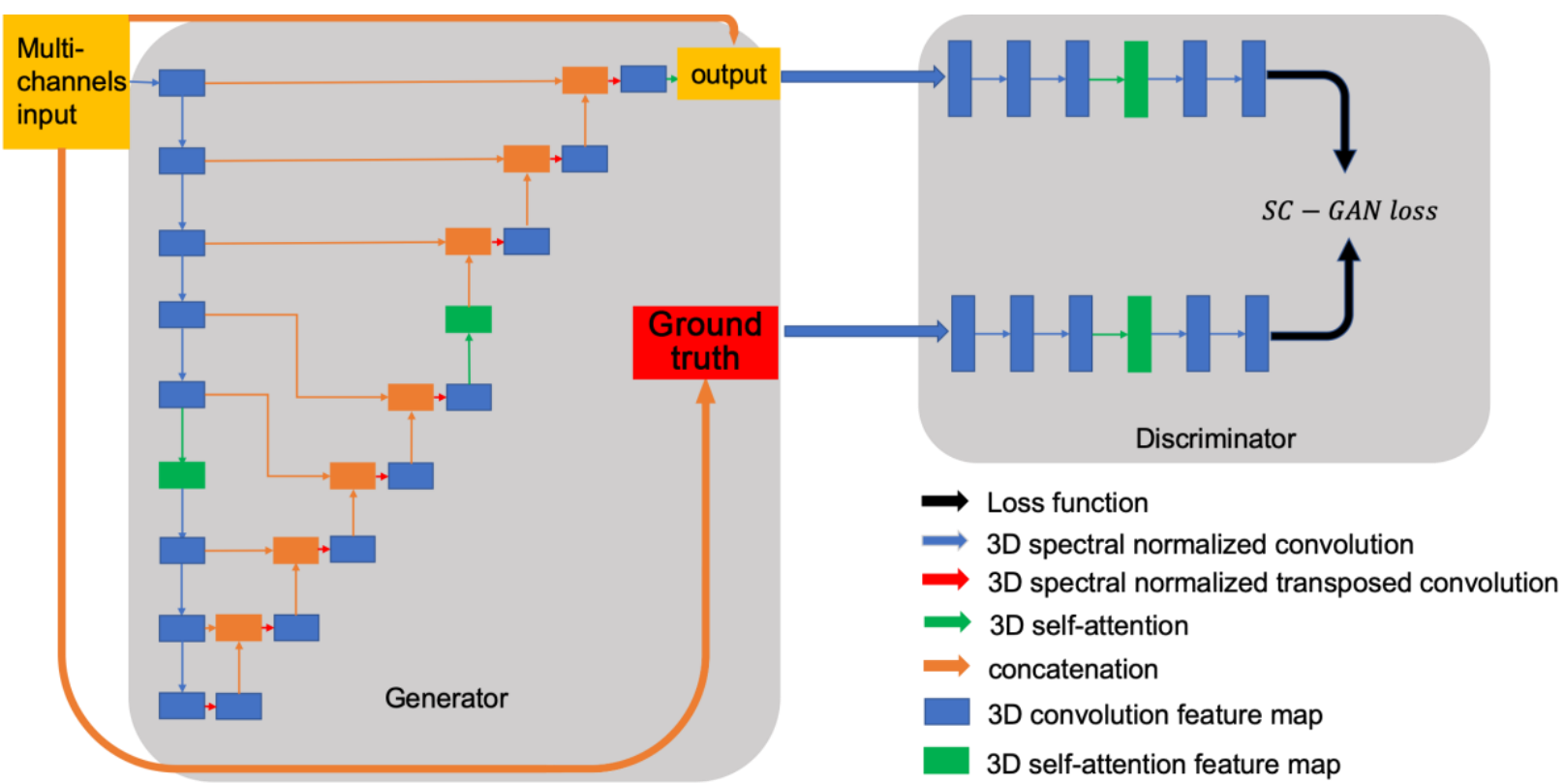

Figure 2. SC-GAN structure with 3D self-attention module. The network structure of SC-GAN constitutes of two parts generator and discriminator. The generator is a Unet like 8 layers encoder-decoder with 3D self-attention module in the middle of encoder and decoder. The discriminator is a 5 layers patch GAN with 3D self-attention. Self-attention module empowers the both generator and discriminator in the adversarial learning strategy. 


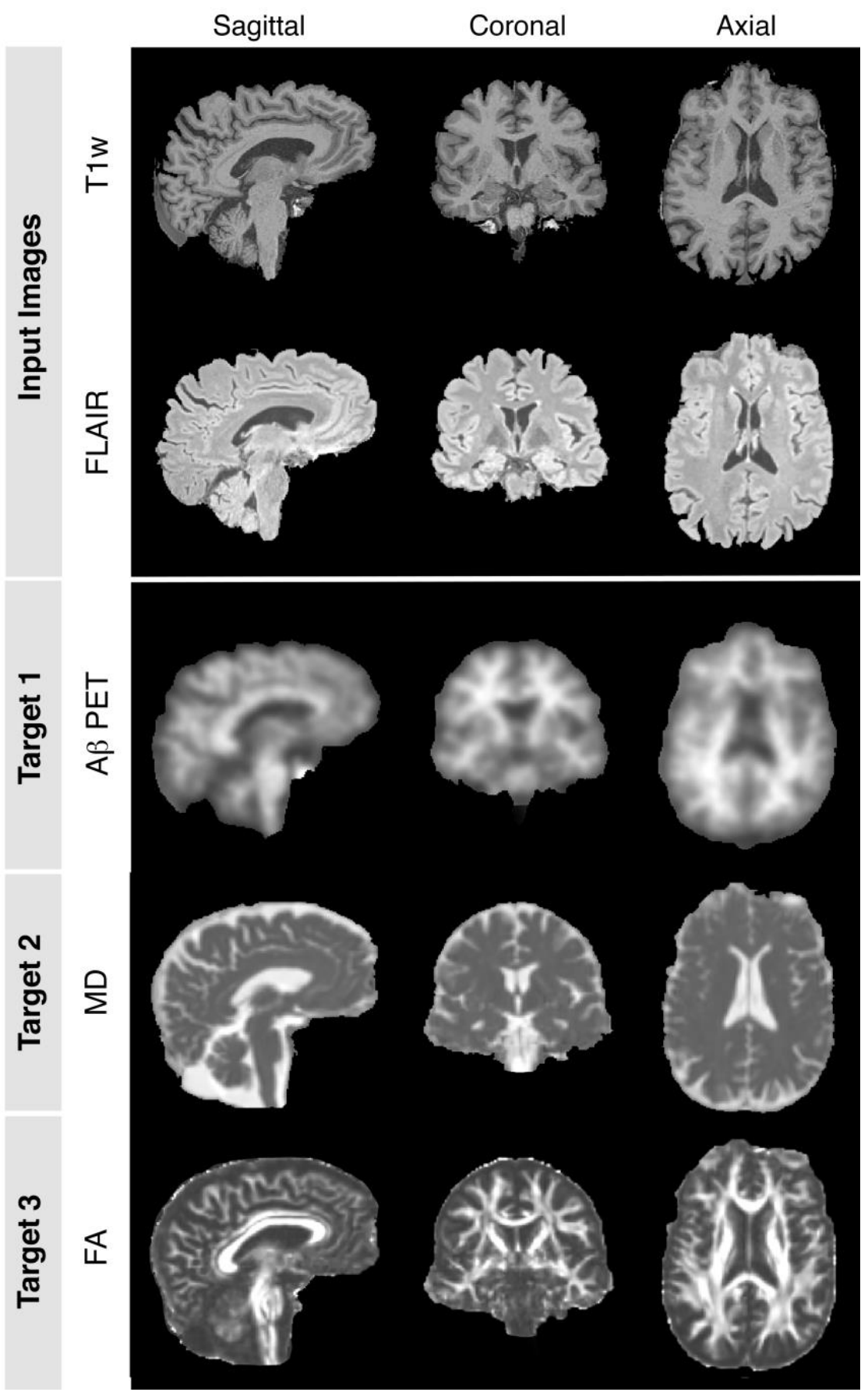

Figure 3. Multi-modal (multi-channel) input. Examples of different neuroimaging data from single individual are presented. T1-weighted (T1w) and fluid-attenuated inversion recovery (FLAIR) were used as input for different synthesis tasks. For each the study tasks a different target was used, which are shown as outputs 1-3: Mean Diffusivity (MD), Fractional Anisotropy (FA) and Amyloid-beta Positron Emission Tomography (A $\beta$-PET). Data were preprocessed and coregistered (see method section for detail), are shown from three anatomical views (from left to right: axial, coronal and sagittal). 

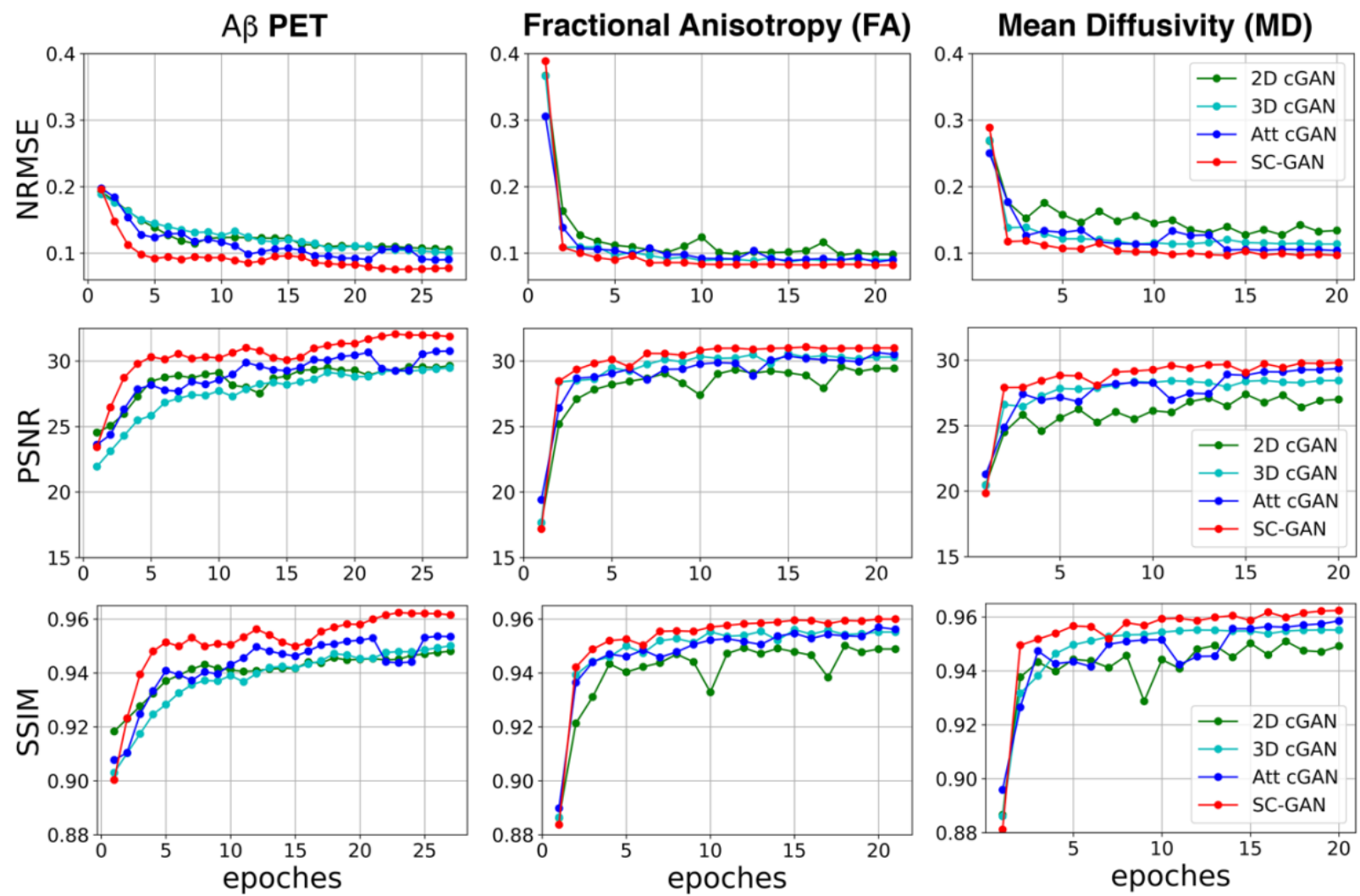

3 Figure 4. Learning curves SC-GAN compared to other Synthesis GANs across different taks. Plots demonstrate learning curves of four CNN networks that were evaluated in this study: 2D GAN, 3D GAN, 3D cGAN with Attention gate (Att cGAN) and SC-GAN. T1w and FLAIR were used for three tasks: 1) synthesizing Amyloid-beta PET ( $n=242$, first column); 2) synthesizing fractional anisotropy ( $n=480$, second column); 3) synthesizing mean diffusivity ( $n=480$, third column).

8 Three different evaluation metrics were used: First row shows normalized root mean square error (NRMSE); Second row shows peak signal-to-noise ratio (PSNR); Third row shows structural similarity (SSIM). Note that all networks reached their plateau around epoch $=20$. 
(a) Ab PET

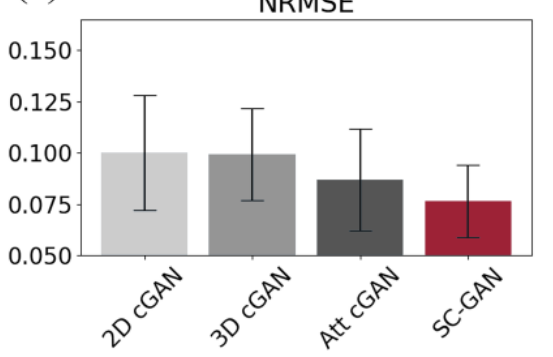

(b) FA

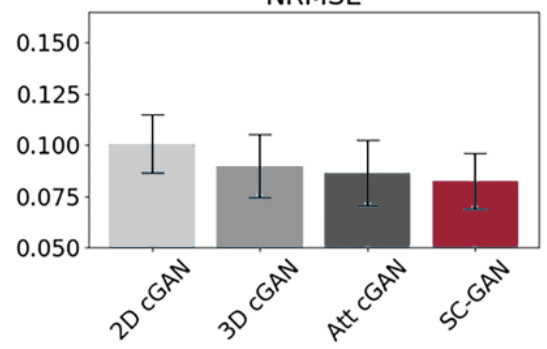

(c) MD

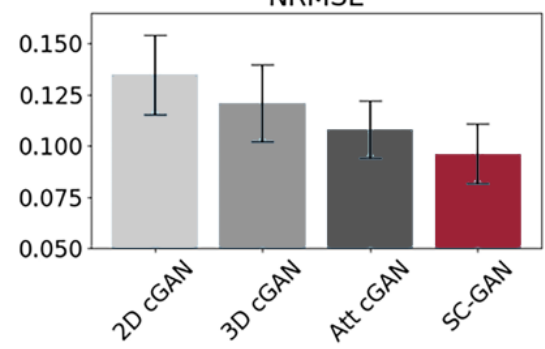

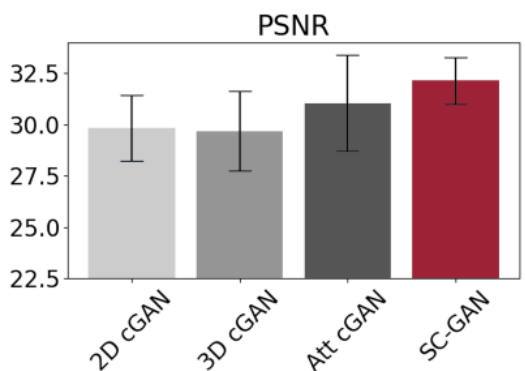

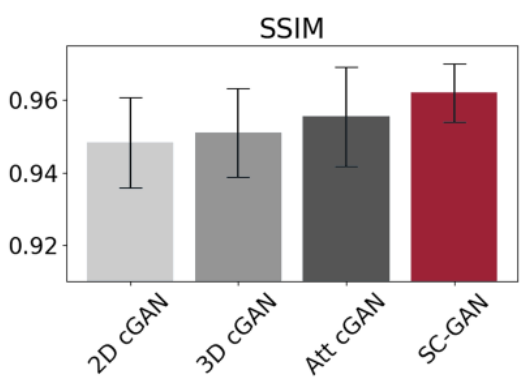

PSNR
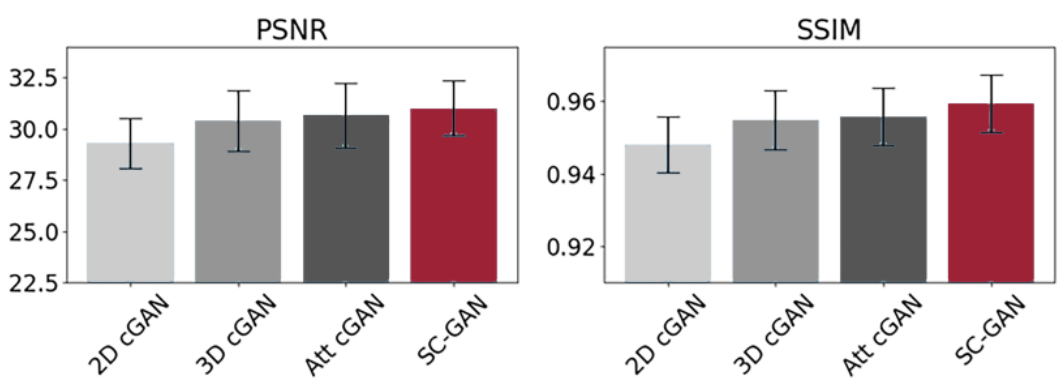

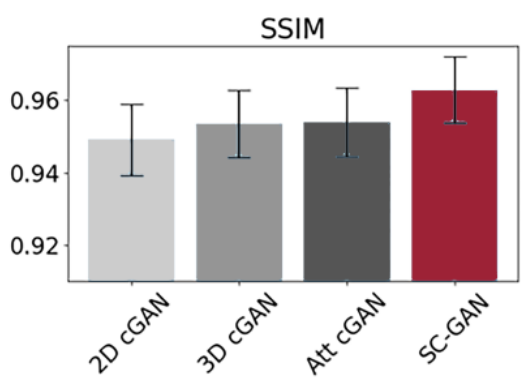

Figure 5. Image quality metrics on test data across different tasks. Bar charts demonstrate normalized mean square error (NRMSE), peak signal-to-noise ratio (PSNR) and structural similarity (SSIM) among test images after the networks reached the plateau and the hyperparameters were optimized. T1w and FLAIR were used for three tasks: 1) synthesizing Amyloid-beta PET ( $n=242, A) ; 2)$ synthesizing fractional anisotropy $(n=480, B) ; 3)$ synthesizing mean diffusivity $(n=480, C)$. 


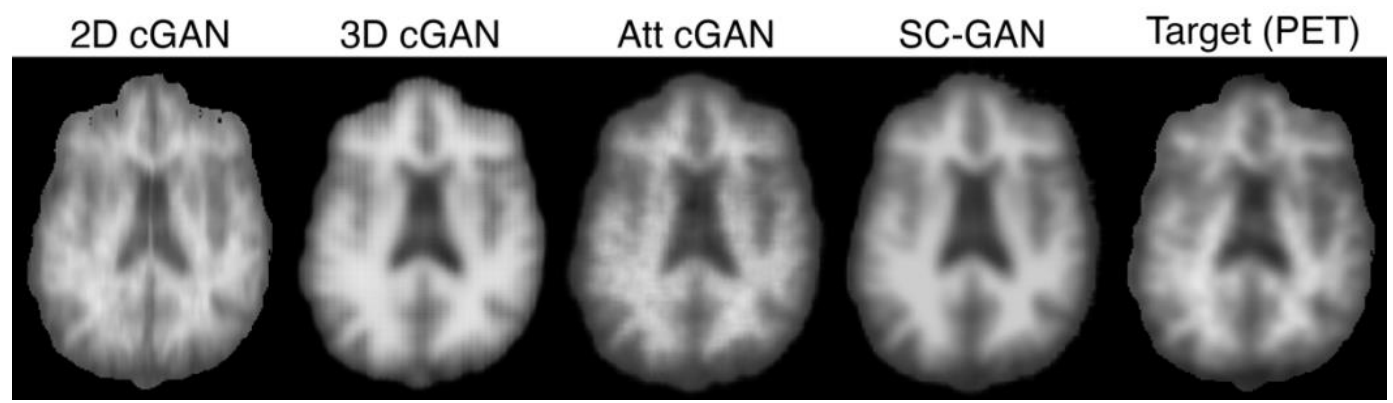

Figure 6. Qualitative assessment of PET synthesis task. Images are results of applying different GANs on T1w and FLAIR input images to predict Amyloid-beta PET. Target PET is also illustrated for comparison. Target image is normalized to [0 1] range for training and an equal color range of [0 1] are used for visualization.

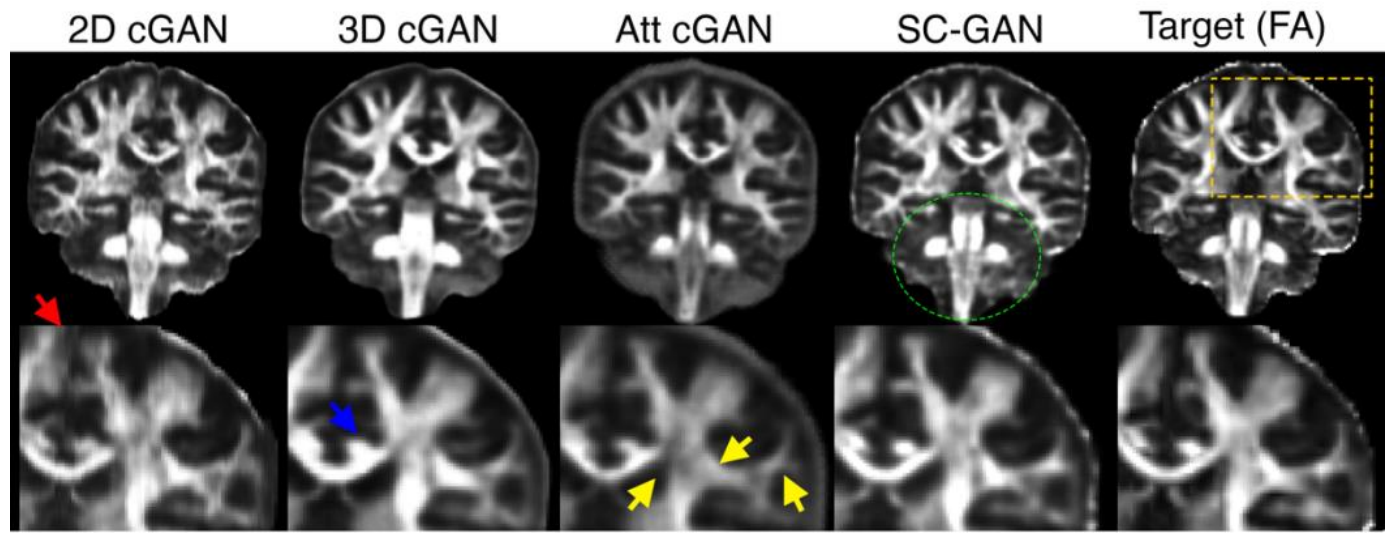

Figure 7. Qualitative assessment of fractional anisotropy (FA) synthesis task. Images are results of applying different GANs on T1w and FLAIR input images to predict FA. Target FA is also illustrated for comparison. An equal color range of [0 1] are used for visualization. Note that SCGAN were able to synthesize FA in more detail in comparison with other networks. The 2D network demonstrated continuous distortion (red arrow), 3D cGAN resulted to an oversmoothed image (see blue arrow showing partial volume effect between fiber bundles of cingulum and corpus callosum). Attention cGAN failed to capture high intensity FA across the white matter (yellow arrows). Green dotted circle shows that, unlike other networks, SC-GAN was able to capture brainstem details. 


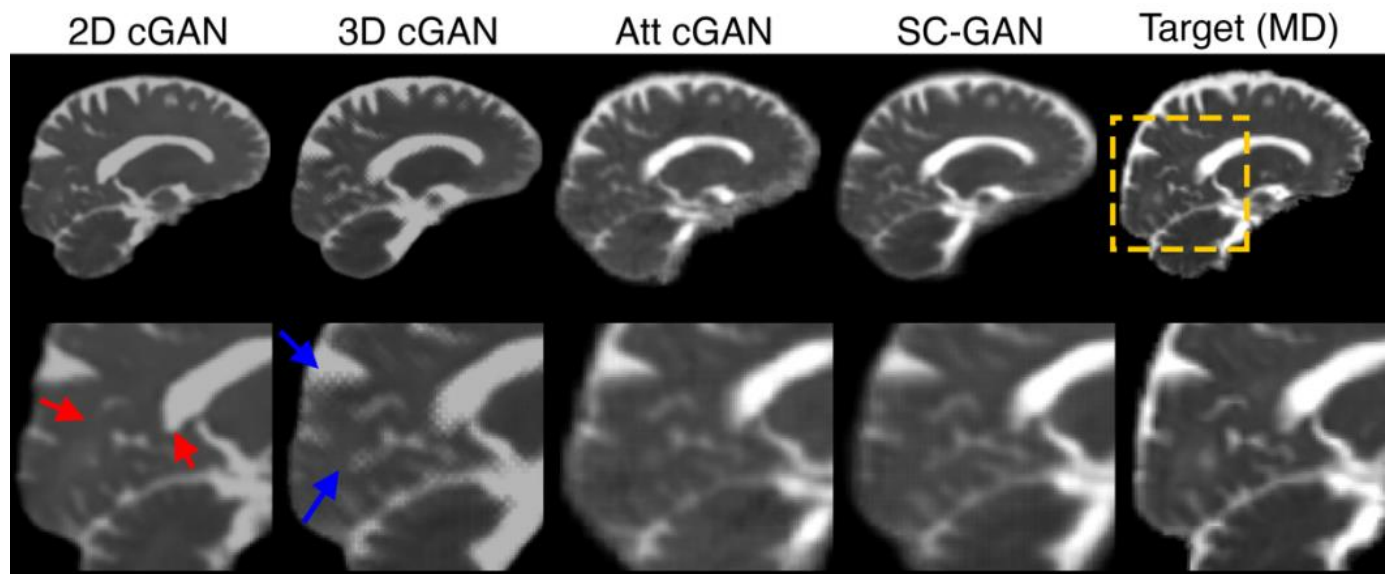

Figure 8. Qualitative assessment of mean diffusivity (MD) synthesis task. Images are results of applying different GANs on T1w and FLAIR input images to predict MD. Target MD is also illustrated for comparison. Target image is normalized to [0 1] range for training and an equal color range of [0 1] are used for visualization. Note that SC-GAN were able to synthesize MD in more detail in comparison with other networks. The 2D generated artificial sharp boundaries (red arrow) and 3D cGAN resulted to a large amount of striping artifact (blue arrow). 
bioRxiv preprint doi: https://doi.org/10.1101/2020.06.09.143297; this version posted June 11,2020. The copyright holder for this preprint (which was not certified by peer review) is the author/funder, who has granted bioRxiv a license to display the preprint in perpetuity. It is made available under aCC-BY-NC-ND 4.0 International license.
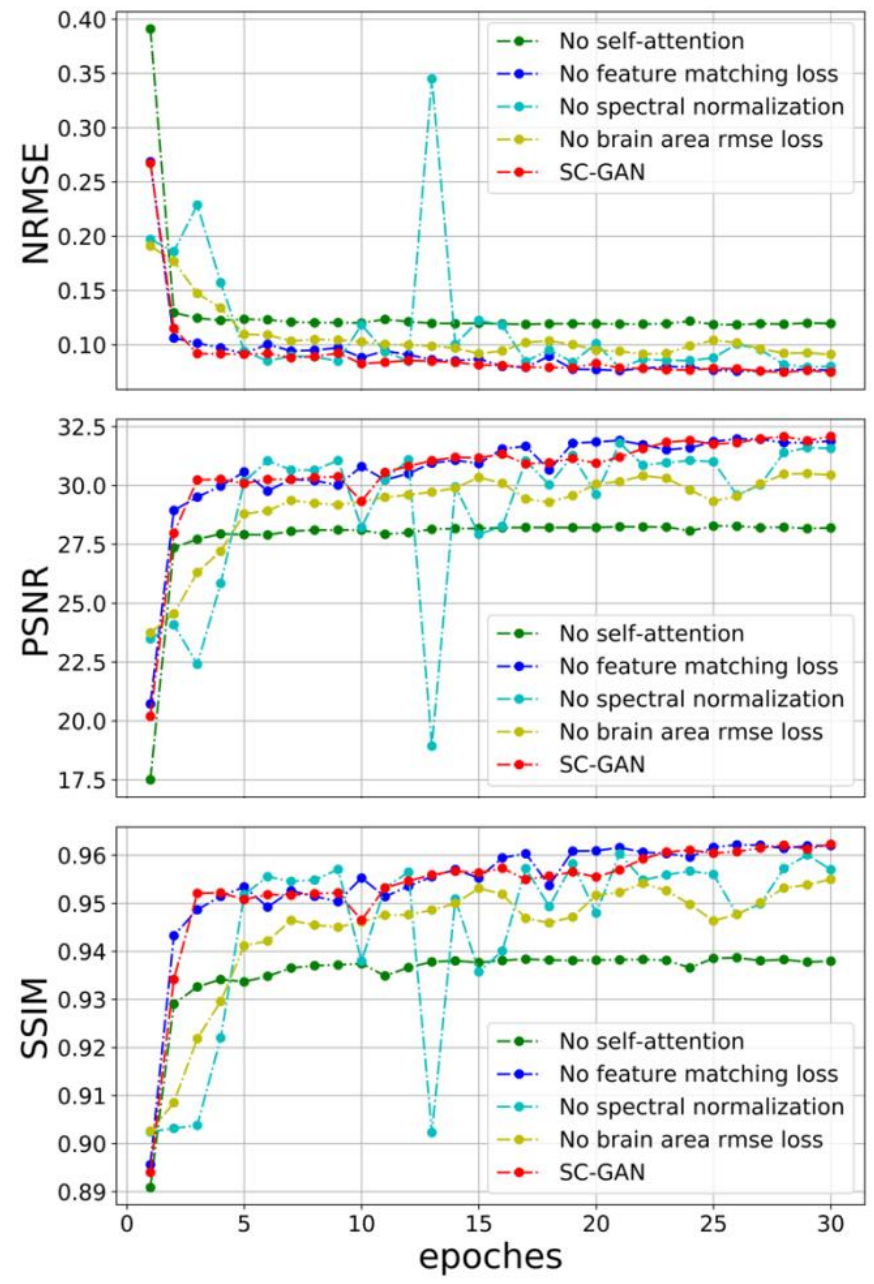

1

Figure 9. Ablation test across modules of SC-GAN. The SC-GAN with and without different network modules were assessed on the $A \beta$ PET synthesis task and learning curves across different evaluation criteria are presented here. Plots demonstrate normalized mean square error (NRMSE), peak signal-to-noise ratio (PSNR) and structural similarity (SSIM). The self-attention module appeared to have the highest contribution to the achieved improvement, followed by spectral normalization and non-brain loss function exclusion. 


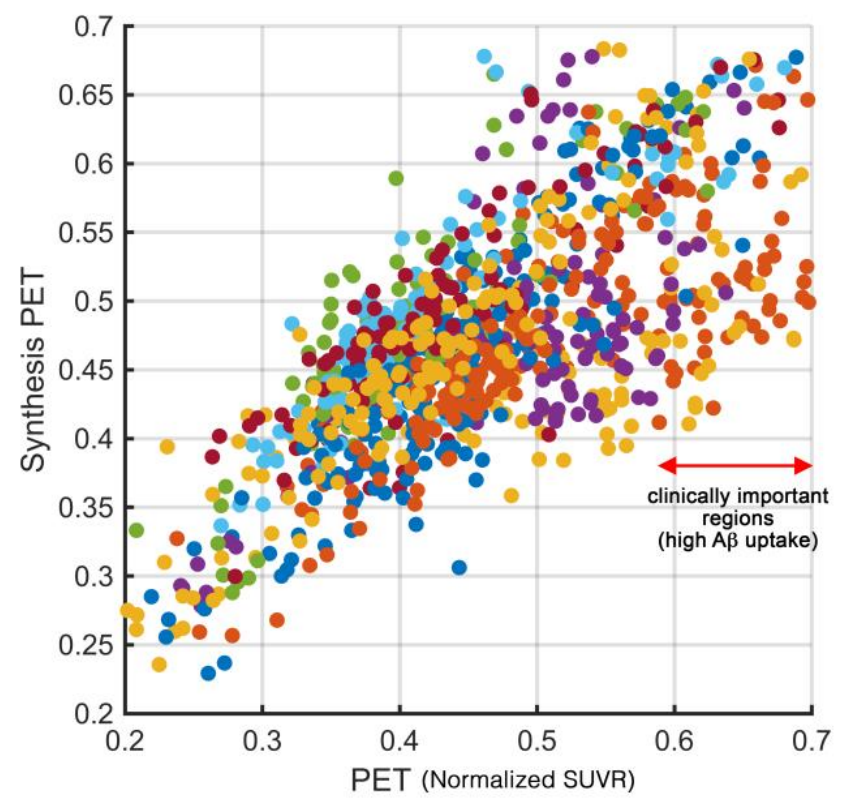

2

11
13
14
16
17
18
19
20
21
23
24

Figure 10. Correlation between PET and synthesis PET. Plot shows the correlation between A $\beta$ standard uptake value ratio (SUVR) across subcortical and cortical regions of ten test participants (each color represents regions of each participants). PET images that were used for training were normalized using min-max normalization approach. Therefore, test PET images were also normalized using the same approach before comparison. Note that on region with high load of $A \beta$ (shown with red arrow), the synthesis error is higher, suggesting that synthesis PET could not substitute PET imaging. 


\begin{tabular}{|c|c|c|c|c|}
\hline $\begin{array}{l}\text { Synthesis } \\
\text { Task (target } \\
\text { image) }\end{array}$ & Method & $\begin{array}{l}\text { NRMSE } \\
\text { mean }( \pm \mathbf{s t d})\end{array}$ & $\begin{array}{l}\text { PSNR } \\
\text { mean }( \pm \text { std })\end{array}$ & $\begin{array}{l}\text { SSIM } \\
\text { mean }( \pm \mathbf{s t d})\end{array}$ \\
\hline \multirow{4}{*}{ PET } & 2D cGAN & $0.100 \pm 0.028$ & $29.80 \pm 1.59$ & $0.948 \pm 0.010$ \\
\hline & 3D cGAN & $0.099 \pm 0.022$ & $29.69 \pm 1.96$ & $0.950 \pm 0.012$ \\
\hline & Att cGAN & $0.086 \pm 0.024$ & $31.03 \pm 2.34$ & $0.955 \pm 0.014$ \\
\hline & SC GAN & $0.076 \pm 0.017$ & $32.14 \pm 1.10$ & $0.962 \pm 0.008$ \\
\hline \multirow{4}{*}{ FA } & 2D cGAN & $0.100 \pm 0.014$ & $29.29 \pm 1.23$ & $0.948 \pm 0.008$ \\
\hline & 3D cGAN & $0.089 \pm 0.015$ & $30.39 \pm 1.47$ & $0.955 \pm 0.008$ \\
\hline & Att cGAN & $0.086 \pm 0.014$ & $30.65 \pm 1.41$ & $0.956 \pm 0.008$ \\
\hline & SC GAN & $0.082 \pm 0.013$ & $31.00 \pm 1.12$ & $0.959 \pm 0.007$ \\
\hline \multirow{4}{*}{ MD } & 2D cGAN & $0.135 \pm 0.019$ & $26.98 \pm 1.38$ & $0.949 \pm 0.010$ \\
\hline & 3D cGAN & $0.121 \pm 0.018$ & $27.93 \pm 1.42$ & $0.953 \pm 0.010$ \\
\hline & Att cGAN & $0.108 \pm 0.014$ & $28.74 \pm 1.19$ & $0.954 \pm 0.009$ \\
\hline & SC GAN & $0.096 \pm 0.014$ & $29.75 \pm 1.25$ & $0.963 \pm 0.009$ \\
\hline
\end{tabular}

1 Table 1. Comparison among different networks. Table shows statistic values of NRMSE, PSNR

2 SSIM among test images after the networks reached the plateau and the hyperparameters were

3 optimized. Statistically significant results are highlighted in bold font.

\begin{tabular}{lccc} 
Ablation study & NRMSE & PSNR & SSIM \\
& mean $( \pm \mathbf{s t d})$ & mean $( \pm \mathbf{s t d})$ & mean $( \pm \mathbf{s t d})$ \\
\hline No self-attention & $0.118 \pm 0.016$ & $28.34 \pm 1.200$ & $0.939 \pm 0.011$ \\
\hline No adversarial learning & $0.102 \pm 0.018$ & $29.72 \pm 1.583$ & $0.947 \pm 0.012$ \\
\hline No brain area rmse loss & $0.092 \pm 0.017$ & $30.27 \pm 1.627$ & $0.953 \pm 0.010$ \\
\hline No spectral normalization & $0.080 \pm 0.017$ & $31.57 \pm 1.203$ & $0.957 \pm 0.010$ \\
\hline No feature matching & $0.078 \pm 0.019$ & $32.03 \pm 1.174$ & $0.960 \pm 0.013$ \\
\hline SC-GAN & $\mathbf{0 . 0 7 6} \pm \mathbf{0 . 0 1 7}$ & $\mathbf{3 2 . 1 4} \pm \mathbf{1 . 1 0 0}$ & $\mathbf{0 . 9 6 2} \pm \mathbf{0 . 0 0 8}$
\end{tabular}

8 Table 2. Ablation study of SC-GAN. Table shows ablation study of different components of SC-

9 GAN on the $A \beta$ PET synthesis task. 